\title{
Slow logarithmic relaxation in models with hierarchically constrained dynamics
}

\author{
J. J. Brey and A. Prados \\ Física Teórica, Universidad de Sevilla, Apartado de Correos 1065, 41080 Sevilla, Spain
}

(Received 14 September 2000; published 24 January 2001)

\begin{abstract}
A general kind of model with hierarchically constrained dynamics is shown to exhibit logarithmic anomalous relaxation similar to a variety of complex strongly interacting materials. The logarithmic behavior describes most of the decay of the response function.
\end{abstract}

DOI: $10.1103 /$ PhysRevE.63.021108

PACS number(s): 82.40.Bj. 05.40.-a, 31.70.Hq

In past years, an anomalous slow relaxation that can be accurately described by a logarithmic law has been found in the time evolution of a variety of complex strongly interacting materials. This includes spin-glasses [1,2], granular materials [3-6], structural glasses [7-9], and protein models $[11,10]$. In all the above cases, the decay of some perturbation $\phi(t)$ can be fitted at intermediate times by an expression of the form

$$
\phi(t)=C_{1}-C_{2} \ln t,
$$

where $C_{1}$ and $C_{2}$ are constants, and may depend on the macroscopic variables (temperature, compactivity, etc.) characterizing the state of the system. The time range in which the logarithmic law accurately describes the relaxation covers most of the change of $\phi(t)$ from its initial value to the final one.

Palmer, Stein, Abrahams, and Anderson (PSAA) in a seminal paper [12] described some dynamical models for relaxation in complex systems with strong interactions. The models were based in a hierarchically constrained dynamics so that the relaxation of the system involves a sequential series of correlated processes. Although PSAA concentrated on those physical systems showing "stretched exponential", relaxation, it is clear that the general picture emerging from hierarchically constrained dynamics can be translated into very different physical situations. As a consequence, the above models provide a very useful tool to investigate many kinds of " anomalous relaxation" in complex systems.

In particular, the picture of hierarchical constrained dynamics seems to be physically appropriate to describe many of the complex systems showing logarithmic relaxation. Let us consider, as a particular example the densification of powders and structural glasses at high hydrostatic pressure studied in Refs. [4,5,7,9]. External pressure causes some selected mesoscopic regions of the system to reach a more compact state very quickly. The nearest neighbor regions are influenced by this rearrangement, and can also reorganize themselves. Of course, these new structural changes will affect the regions outside the nearest neighbors, allowing them to relax, and so on. The above discussion suggests that the relaxation in this kind of system is slow because it consists of a large number of correlated reorganization steps with an increasing characteristic time. In other words, it has a hierarchical structure, with the faster degrees of freedom (associated with the "inner' regions) controlling the relaxation of the slower degrees of freedom (associated with the "outer", regions). A similar picture has been already suggested in Refs. [4,5]. The aim of this paper is to introduce a quite general class of hierarchical models, which seem appropriate to describe this kind of system, in order to derive the logarithmic relaxation of Eq. (1).

Like PSAA, we will formulate our model in the most general way, hoping it characterizes the behavior of a specific class of systems. We assume that the state of the system can be given by means of $N$ modes. They do not correspond to a particle description of the system, but to some mesoscopic level of description. In this sense, $N$ does not scale with the size of the system, but it is assumed to have a definite value, independent of the size, for a large enough (macroscopic) system. Of course, the specific value depends on the system and the physical situation we are dealing with.

The $N$ modes can be grouped into a discrete series of levels, $n=0,1,2, \ldots$. The number of modes belonging to level $n$ will be denoted by $N_{n}$, so that $N=\Sigma_{n} N_{n}$. Moreover, modes in level $n$ will be indicated by the variables $S_{i}^{(n)}, i$ $=1,2, \ldots, N_{n}$. For the densification experiments of Refs. $[4,5,7,9]$, we can regard $S_{i}^{(n)}$ as a pseudospin variable, with its two possible values corresponding to different volumes of the mesoscopic regions. The dynamics of the system is defined in a hierarchical way as follows. A mode in level $n$ +1 can only modify its state if the configuration of modes in level $n$ belongs to a well-defined subset of all the possible configurations of the modes in that level. Clearly, this slows down the modes in a given level, as compared with those in the previous level. More specifically, the average transition rate $W_{n+1}$ for a mode in level $n+1$ is assumed to have the form [12]

$$
W_{n+1}=W_{n} P_{n}
$$

where $P_{n}$ is the probability that the modes in level $n$ are in any of the configurations allowing us to relax level $n+1$. Our choice for $P_{n}$ will be

$$
P_{n}=\exp \left(-N_{n} \Delta \mu\right)
$$

with $\Delta \mu$ being a dimensionless free energy defining the activation barrier per mode. Equation (3) appears quite naturally if $P_{n}$ is evaluated from the equilibrium distribution of the system under consideration, provided that the subset of configurations of level $n$ allowing us to relax level $n+1$ involves all the modes (or an extensive part of them) in level $n$. Of course, the free energy $\Delta \mu$ must be appropriately in- 
terpreted in each particular situation. For instance, in the densification experiments at high pressure $[4,5,7,9]$ it would be an activation enthalpy divided by the temperature of the sample. On the other hand, in a granular system, it could be understood as an "effective volume" divided by the compactivity [13-16]. Thus, the corresponding average relaxation time of the mode is

$$
\tau_{n+1}=\tau_{n} \exp \left(N_{n} \Delta \mu\right) .
$$

For the sake of simplicity, the barrier $\Delta \mu$ is assumed to be independent of the level $n$ of the mode and also of the number of modes in the level $N_{n}$. Iteration of Eq. (4) yields

$$
\tau_{n}=\tau_{0} \exp \left(\Delta \mu \sum_{m=0}^{n-1} N_{m}\right)
$$

The range of variation of $n$ and the values of the populations $N_{n}$ characterize the complexity of the time evolution of the system. In principle, there is no need for a maximum value $n_{\max }$ of $n$, i.e., $n_{\max }$ may be infinite. On the other hand, the systems we are interested in show slow relaxation, which requires a large relaxation time. In this way, it is necessary that

$$
\tau_{\max }=\lim _{n \rightarrow n_{\max }} \tau_{n} \gg \tau_{0} .
$$

This condition for slow relaxation was already pointed out by PSAA [12].

We are interested in the relaxation of the quantity

$$
Q(t)=\frac{1}{N} \sum_{n} \sum_{i=1}^{N_{n}}\left\langle S_{i}^{(n)}(t)\right\rangle,
$$

where the angular brackets denote configuration average. Note that in the context of the densification experiments, $[4,5,7,9], Q(t)$ would be proportional to the actual volume of the sample at time $t$, with the physical meaning given above to the variables $S_{i}^{(n)}$. The corresponding relaxation function is defined in terms of the asymptotic long-time value $Q(\infty)$ as

$$
\phi(t)=\frac{Q(t)-Q(\infty)}{Q(0)-Q(\infty)}=\sum_{n} \omega_{n} \exp \left(-\frac{t}{\tau_{n}}\right),
$$

with $\omega_{n}=N_{n} / N$ being the fraction of modes in level $n$. Combination of Eqs. (5) and (8) gives

$$
\phi(t)=\sum_{n=0}^{n=n_{\max }} \omega_{n} \exp \left[-\frac{t}{\tau_{0}} \exp \left(-\zeta \sum_{m=0}^{n-1} \omega_{m}\right)\right],
$$

where

$$
\zeta=N \Delta \mu .
$$

Next we will assume that $\omega_{n}$ changes very smoothly with $n$. This allows us to introduce a continuum limit for the distribution of relaxation times, which is expected to be closer to real systems than the discrete level model considered up to now. We define a function $\tilde{\omega}(x)$ by $\tilde{\omega}(n \epsilon)=\omega_{n}$, with $\epsilon$ $\ll 1$, so that the variable $x_{n}=n \epsilon$ is almost continuum and the sum over $n$ can be replaced by integrals. Let us introduce a new variable $x_{\max }=n_{\max } \epsilon$

$$
u=\frac{\int_{0}^{x} d x^{\prime} \tilde{\omega}\left(x^{\prime}\right)}{\int_{0}^{x_{\max }} d x^{\prime} \tilde{\omega}\left(x^{\prime}\right)},
$$

$0 \leqslant u \leqslant 1$, which measures the fraction of the total number of modes $N$ that are in levels up to a value $n=x / \epsilon$. It is then a simple task to transform Eq. (9) into

$$
\phi(t)=\int_{0}^{1} d u \exp \left(-\frac{t}{\tau_{0}} e^{-\zeta u}\right)
$$

showing that the decay of $\phi$ can be characterized by only two parameters, namely, $\tau_{0}$ and $\zeta$. The former fixes the time scale, while the latter determines the actual shape of the relaxation. The elementary relaxation times given by Eq. (5) are $\tau(u)=\tau_{0} \exp (\zeta u)$ and, in particular, $\tau_{\max }=\tau_{0} \exp (\zeta)$. Thus, the condition for slow relaxation, Eq. (6), implies that $\exp (\zeta) \gg 1$.

The analysis of Eq. (12) is greatly simplified by realizing that it is equivalent to

$$
\phi(t)=\frac{1}{\zeta}\left[E_{1}\left(\frac{t}{\tau_{0}} e^{-\zeta}\right)-E_{1}\left(\frac{t}{\tau_{0}}\right)\right],
$$

where $E_{1}(z)$ is the integral exponential function [17]. There are two natural time scales in Eq. (13). One is defined by $t / \tau_{0}$, while the other is a slow time scale defined by $s$ $=\left(t / \tau_{0}\right) e^{-\zeta} \ll\left(t / \tau_{0}\right)$. In the following, we will use the asymptotic expansions of the exponential integral function from Ref. [17]. For very short times such that $t / \tau_{0} \ll 1$, Eq. (13) is equal to

$$
\phi(t)=1-\frac{1-e^{-\zeta}}{\zeta} \frac{t}{t_{0}}+O\left(\frac{t}{\tau_{0}}\right)^{2}
$$

i.e., $\phi(t)$ decays very little from its initial unity value in this region. In the opposite limit of very large times for which $s \gg 1$, it is

$$
\phi(t) \sim \frac{1}{\zeta} \frac{e^{-s}}{s},
$$

the response function has already decayed to very small values. One main conclusion of the above discussion is that the relevant part of the relaxation, in which $\phi(t)$ changes from values very close to unity to very close to zero, takes place between the two asymptotic time regimes we have analyzed. In this way, we are led to consider the distinguished limit $t / \tau_{0} \gg 1, s \ll 1$. In this intermediate time window it is found that 


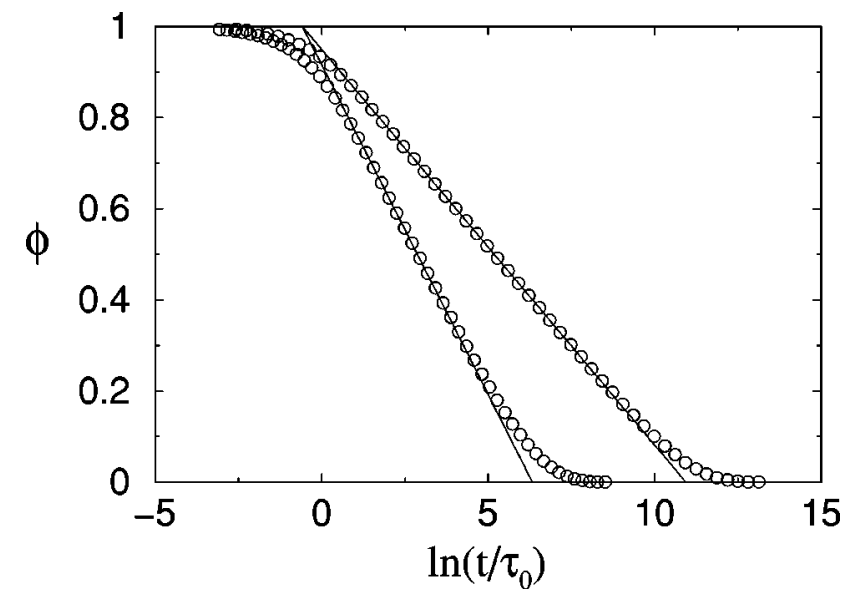

FIG. 1. Relaxation function $\phi$ plotted as a function of time. From left to right, the curves correspond to $\exp (\zeta)=10^{3}$ and $10^{5}$. The integral representation of $\phi$ [Eq. (13)], is given by the circles, while the solid lines are the asymptotic expression for intermediate times [Eq. (16)].

$$
\phi(t) \sim 1-\frac{1}{\zeta}\left(\gamma+\ln \frac{t}{\tau_{0}}\right) .
$$

This is precisely the logarithmic decay of Eq. (1). Moreover, the previous discussion of the short- and large-time limits clearly indicates that most of relaxation of the system is expected to be accurately described by Eq. (16). As an example, we present in Fig. 1 the relaxation function $\phi(t)$ for $\exp (\zeta)=10^{3}$ and $\exp (\zeta)=10^{5}$. The integral expression given by Eq. (12) (circles) is well fitted by the logarithmic asymptotic form of Eq. (16) (solid lines). The agreement extends over several decades of intermediate times, measured in units of the basic time $\tau_{0}$. The fit improves as the value of $\zeta$ increases, consistently with the condition for time scales separation.

It is also interesting to compute an average relaxation time $\bar{\tau}$, given by

$$
\bar{\tau}=\int_{0}^{\infty} d t \phi(t)=\tau_{0} \frac{e^{\zeta}-1}{\zeta} \sim \tau_{0} \frac{e^{\zeta}}{\zeta},
$$

showing an exponential increase with $\zeta$. Taking into account the definition of $\zeta$ [Eq. (10)] the above result can be understood as corresponding to an activation energy barrier whose height is proportional to the complexity of the system, measured by the total number of modes $N$ actively involved in the relaxation. As already mentioned, for a given specific situation, the dimensionless parameter $\Delta \mu$ might have a well-defined physical interpretation, being, for instance, the ratio between the Gibbs free energy barrier per mode and the thermal energy. In this way, it is also interesting to note that the coefficient of $\ln t$ in Eq. (16) is $\zeta^{-1}$, which is then proportional to the temperature $T$. This would lead to the $T \ln t$ behavior observed in many physical systems, for instance, in Refs. [2,9].

In summary, we have studied a simple class of models that exhibit an anomalous logarithmic relaxation. The dynamics is hierarchically constrained and is adequate to study slow relaxation because there is a clear separation of time scales. The slow logarithmic relaxation is similar to what is experimentally observed in a variety of complex systems; fitting the data in most of the relevant part of the relaxation, i.e., the time window where the relaxation function is neither too small nor too close to its initial value. Perhaps the main assumption of this work is the "extensiveness" with $N_{n}$ of the effective barrier for modes in level $n+1$, as expressed by Eq. (3). This is quite a general condition and, therefore, the class of hierarchical models presented here may provide an explanation of the appearance of linear logarithmic relaxation in very different physical situations. In this context, the role of hierarchically constrained dynamics may also be relevant in order to understand other kinds of anomalous relaxation, as the inverse logarithmic behavior found in compaction experiments of granular systems $[18,19]$. Also, it seems interesting to investigate the behavior of hierarchical models in the temperature cycling experiments usually carried out in glassy systems [20].

This research was partially supported by Grant No. PB981124 from the Dirección General de Investigación Científica y Técnica (Spain).
[1] P. Nordblad, P. Svedlindh, L. Lundgren, and L. Sandlund, Phys. Rev. B 33, 645 (1986).

[2] E. I. Shakhnovich and A. M. Gutin, Europhys. Lett. 9, 569 (1989).

[3] H. M. Jaeger, C.-h. Liu, and S. R. Nagel, Phys. Rev. Lett. 62, 40 (1989).

[4] V. V. Bredikhin, V. A. Sidorov, O. B. Tsiok, E. V. Gurovich, and L. G. Khvostantsev, Europhys. Lett. 18, 111 (1992).

[5] O. B. Tsiok, V. A. Sidorov, V. V. Bredikhin, L. G. Khvostantsev, V. N. Troitskiy, and L. I. Trusov, Phys. Rev. B 51, 12127 (1995).

[6] A. Mehta, R. J. Needs, and S. Dattagupta, J. Stat. Phys. 68, 1131 (1992).

[7] O. B. Tsiok, V. Brazhkin, A. G. Lyapin, and L. G. Khovstant- sev, Phys. Rev. Lett. 80, 999 (1998).

[8] L. Fabbian, W. Götze, F. Sciotino, P. Tartaglia, and F. Thiery, Phys. Rev. E 59, R1347 (1999).

[9] V. G. Karpov and M. Grimsditch, Phys. Rev. B 48, 6941 (1993).

[10] M. Skorobogatiy, H. Guo, and M. Zuckermann, J. Chem. Phys. 109, 2528 (1998).

[11] A. Fernández and G. Appignanesi, Phys. Rev. Lett. 78, 2668 (1997).

[12] R. G. Palmer, D. L. Stein, E. Abrahams, and P. W. Anderson, Phys. Rev. Lett. 53, 958 (1984).

[13] S. F. Edwards and R. B. S. Oakeshott, Physica A 157, 1080 (1989).

[14] A. Mehta and S. F. Edwards, Physica A 157, 1091 (1989). 
[15] J. J. Brey, A. Prados, and B. Sánchez-Rey, Physica A 275, 310 (2000).

[16] A. Prados, J. J. Brey, and B. Sánchez-Rey, Physica A 284, 277 (2000).

[17] Handbook of Mathematical Functions, edited by M. Abramowitz and I. A. Stegun (Dover, New York, 1965).
[18] J. B. Knight, C. G. Frandich, C. N. Lau, H. M. Jaeger, and S. R. Nagel, Phys. Rev. E 51, 3957 (1995).

[19] E. R. Nowak, J. B. Knight, E. Ben-Naim, H. M. Jaeger, and S. R. Nagel, Phys. Rev. E 57, 1971 (1998).

[20] A. Prados and J. J. Brey (unpublished). 